\title{
Driver's Stress Reaction Times Features when Driving on Urban Road
}

\author{
Cuicui Gao ${ }^{1, a}$, Xiaohui Yuan ${ }^{2, b}$ Pengcheng $\mathrm{Yu}^{2, \mathrm{c}}$ \\ ${ }^{1}$ Department of automobile application engineering, Yantai Automobile Engineering Professional \\ College \\ ${ }^{2}$ School of Automobile, Chang'an University \\ a358210040@qq.com, b1129253586@qq.com, ${ }^{c} 327054359 @ q q . c o m$
}

Keywords: reaction time, urban road, driving simulation, traffic safety.

\begin{abstract}
A driving simulator was set up for analyzing driver's reaction time when driving on urban road environment. 28 participate drivers were recruited for the simulated driving test, each driver's reaction perception time, decision-making time and operation time were measured and analyzed. Results show that the average perception time of experienced drivers $(181.37 \mathrm{~ms})$ is shorter than that of inexperienced drivers (195.62 ms). While experienced driver's decision-making time is longer than that of inexperienced drivers, but more stable. The average operation time of experienced drivers (281.85 ms) are longer than inexperienced drivers (234.3 ms).
\end{abstract}

\section{Introduction}

The drivers' stress reaction behavior includes stress reaction perception, decision making and operation $^{[1]}$. The experience, physiological status and psychological status of the driver can affect stress reaction time ${ }^{[2]}$. Considering that every driver has his or her own character which can deeply affect self-cognition and reaction behavior, different drivers have different stress reaction time even in the same type of accident. Some drivers can perceive danger timely, enabling them to take action to avoid the perceived danger. However, the stress reaction time of some drivers is extremely long, causing them to miss the best time to take action. The urban road traffic environment is very special because of pedestrians, non-motorized vehicles and other vehicles may not comply with the traffic rules frequently ${ }^{[3]}$. This study utilizes virtual reality techniques to develop a set of urban road environment scenes in which the stress reaction time features of several drivers were analyzed through a simulation.

\section{Stress Reaction Experiment}

Stress Reaction Experiment Scenes. More than 10 urban road traffic scenes were designed and developed to identify the characteristics of urban traffic environment. The stress reaction time data of four scenes were selected based on the analysis of common accident data for analysis after the experiment. The adaptability of the driver was considered fully during the design process of the stress scenes to completely immerse the driver in the simulation environment. A long and stable driving process was established before danger set in.

Scene 1: A child appears suddenly from the right to cross the road.

Scene 2: A pedestrian suddenly appears in front of a bus just when the driver of the bus decides to overtake from the left.

Scene 3: A pedestrian changes his walking direction and ends up in front of a car.

Scene 4: At a crossroad under construction, another car suddenly appears from a hidden intersection.

Stress Reaction Simulation System. The stress reaction simulation system of independent research and development was utilized in the experiment to collect the braking and steering data of drivers under stress. The system, which is mainly based on the driver's operational process, shows dynamic 3D traffic stress scenes at real-time. The operational data of the accelerator pedal, brake pedal and steering wheel were collected. The stress reaction simulation system consists mainly of the 
control cabin, data collection device, data processing device, scene simulation system, display platform and sound device. An eye tracker was utilized in the experiment to track the driver's fixation behavior in real-time. The video was played frame by frame to identify the driver's fixation target.

Drivers. All the recruited drivers were willing to participate. The drivers belong to different driving groups to adhere to the diversity principle. Some of the participants are professional, non-professional, experienced, inexperienced, young and old drivers. With regard to driving experience, the least amount of experience is one month and the maximum is 27 years. The average number of driving years is 13 , and the standard deviation is 8.37 years. Twenty-eight drivers were subjected to a test, and the results show that all the drivers can drive cars independently and safely. The drivers have no history of cardiovascular and physiological diseases, and their vision acuity (including corrected vision) is normally.

Experimental Procedures. Before the experiment, the drivers were asked to drive in a traffic scene without danger with the simulation system until the driver is sure that he can operate the system easily and freely. This procedure was performed to familiarize the drivers with the accelerator pedal, brake pedal and steering wheel of the driving simulator.

The drivers' stress reaction process consists of stress reaction perception, decision-making and operation time. The time period from the moment danger appears until the driver identifies the danger signal is defined as perception time ${ }^{[4]}$. The object fixed by the driver's eye and its position as well as the time can be found in every frame of the eye movement video. Decision-making time data were mainly acquired based on the eye movement data collected by the eye tracker and the accelerator pedal data collected by the stress reaction simulation system. The time from the driver relaxes the accelerator pedal to the moment steps on the brake pedal is defined as operation time. Operation time data was mainly acquired based on the accelerator and brake pedal data collected by the stress reaction simulation system.

\section{Experiment Results Analysis}

The drivers were classified into experienced and inexperienced drivers based on the mileage traveled ${ }^{[5]}$. Drivers who traveled more than 50,000 kilometers were classified as experienced drivers, whereas those who traveled less than 50,000 kilometers were classified as inexperienced drivers. Each group's reaction, decision-making and operating time were analyzed, and the results are shown below.

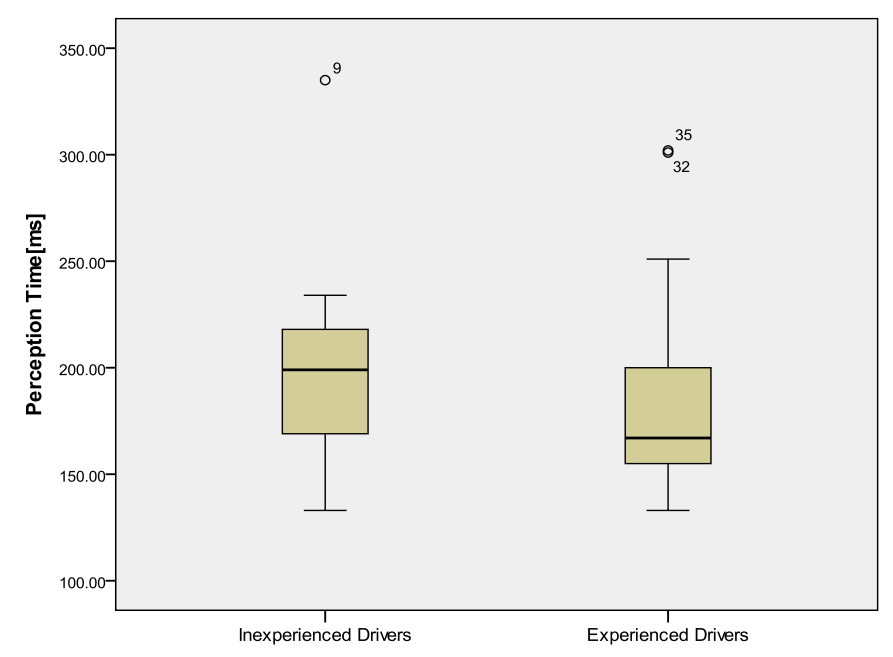

Figure. 1 Perception time of experienced inexperienced drivers. 


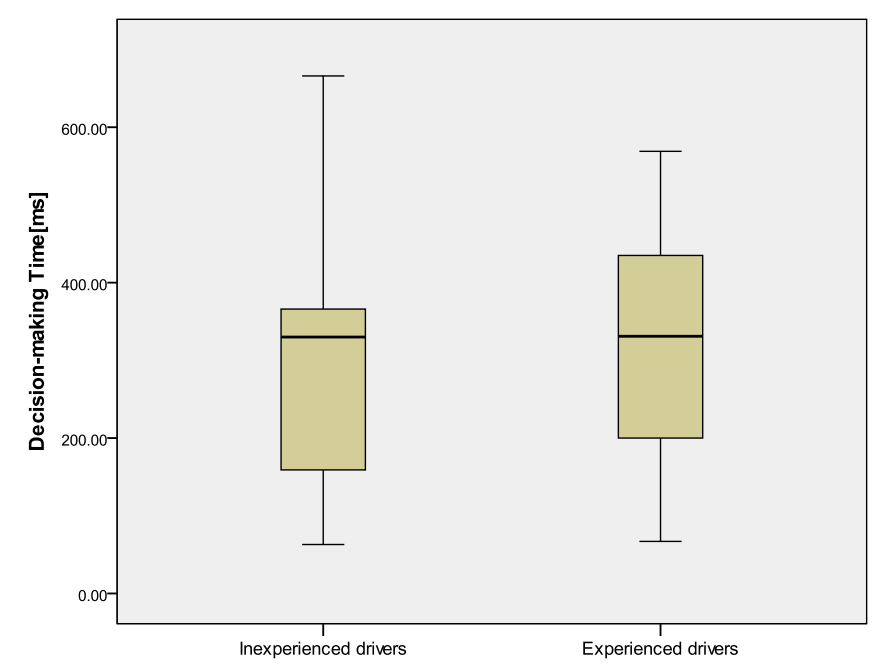

Figure. 2 Decision-making time of and experienced and inexperienced drivers.

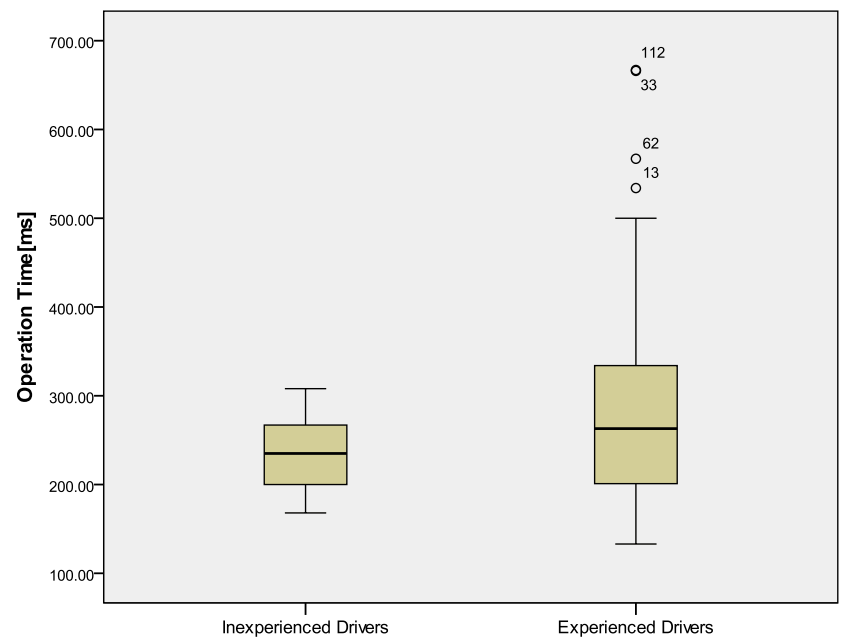

Figure. 3 Operation time of experienced and inexperienced drivers.

Fig. 1 shows that the perception time of the experienced drivers ranges from $133 \mathrm{~ms}$ to $302 \mathrm{~ms}$ with a mean of $181.37 \mathrm{~ms}$ and standard deviation of $42.32 \mathrm{~ms}$. The perception time of the inexperienced drivers ranges from $133 \mathrm{~ms}$ to $335 \mathrm{~ms}$ with a mean of $195.62 \mathrm{~ms}$ and standard deviation of $52.27 \mathrm{~ms}$. The experienced drivers generally perceive danger signals faster than the inexperienced drivers. The average perception time is reduced by approximately $7.8 \%$, and standard deviation is decreased by $23.5 \%$. The ability of the experienced drivers to perceive danger signals is better and more stable than that of the inexperienced drivers. The reason is that experienced drivers have encountered more stress scenes than inexperienced drivers. The experienced drivers have accumulated knowledge on the emergence of danger signals, which help drivers perceive danger quickly.

Fig. 2 shows that because of the differences in the individual characteristics of the drivers the decision-making time of the experienced and inexperienced drivers are significantly different. The decision-making time of the experienced drivers ranges from $67 \mathrm{~ms}$ to $569 \mathrm{~ms}$ with a range ability of $502 \mathrm{~ms}$, whereas the decision-making time of the inexperienced drivers ranges from $63 \mathrm{~ms}$ to $666 \mathrm{~ms}$ with a range ability of 603 ms. However, the standard deviation in the decision-making time of the experienced drivers is $140.33 \mathrm{~ms}$, whereas that of the inexperienced drivers' is $186.03 \mathrm{~ms}$. The value of the former is $32.57 \%$ less than that of the latter. This result shows that the experienced drivers exhibit better and more stable performance than the inexperienced drivers in terms of handling danger. The reason is that the experienced drivers have encountered more driving stress scenes than 
the inexperienced drivers. The experienced drivers have accumulated knowledge on how to handle danger signals, which help drivers respond to danger quickly.

Fig. 3 shows that the operation time of the experienced drivers is significantly different and ranges from $133 \mathrm{~ms}$ to $667 \mathrm{~ms}$ with a range ability of $534 \mathrm{~ms}$. The operation time of the inexperienced drivers ranges from $168 \mathrm{~ms}$ to $308 \mathrm{~ms}$ with a range ability of $140 \mathrm{~ms}$. The standard deviation in the operation time of the experienced drivers is $104.11 \mathrm{~ms}$, whereas that of the inexperienced drivers is $39.88 \mathrm{~ms}$. The value of the former is $161.1 \%$ higher than that of the latter.

This result shows that the inexperienced drivers avoid danger more quickly and decisively than the experienced drivers. The mean operation time of the experienced drivers is $281.85 \mathrm{~ms}$, whereas that of the inexperienced drivers is $234.3 \mathrm{~ms}$. After the inexperienced drivers have decided how to deal with or avoid danger, they operated the vehicle more quickly than the experienced drivers. The operation time of the former is $20.3 \%$ less than that of the experienced drivers. The experienced drivers have longer operation time than the inexperienced drivers because most of the inexperienced drivers are young (aged 20 to 30), whereas most of the experienced drivers are middle-aged (aged 40 to 50). The latter has less agility and flexibility in terms of vehicle operation. Moreover, after the experienced drivers have made optimal decisions on how to avoid collision, they handle possible collision more gently than the inexperienced drivers who often step on the brake pedal completely for emergency braking.

\section{Summary}

This study investigated driver's stress reaction time. Analyses of the samples revealed the findings below.

(1) The perception time of the experienced drivers is shorter than that of the inexperienced drivers.

(2) The decision-making time of the experienced drivers is longer than that of the inexperienced drivers because the former considers danger more comprehensively than the latter.

(3) The operation time of the experienced drivers is longer than that of the inexperienced drivers.

\section{References}

[1] Davoodi Seyed Rasoul, Hamid Hussain, Pazhouhanfar Mahdieh, and Muttart Jeffrey W. (2011). Motorcyclist Perception Response Time in Stopping Sight Distance Situations, Safety Science, n.3, pp.371-377.

[2] Genya Abe, John Richardson (2006). Alarm timing, trust and driver expectation for forward collision warning systems, Applied Ergonomics, n.5, pp.577-586.

[3] José Reynaldo Setti, Hesham Rakha and Ihab El-Shawarby (2006). Analysis of Brake Perception-Reaction Times on High-Speed Signalized Intersection Approaches. Proceedings of the IEEE ITSC 2006, Toronto, Canada.

[4] Paul L. Olson (1986). Perception-Response Time to Unexpected Roadway Hazards, Human Factors, n.1, pp.91-96.

[5] Sumida, Y, Suzuki, H., H. Makishita and K. Matsunaga (2004). A study of automobile drivers' braking reaction times according to age bracket, Trans. Automot. Eng. Jpn., n.3, pp.209-214. 